Research Paper

\title{
Prognostic value of reduced E-cadherin expression in breast cancer: a meta-analysis
}

\author{
Zhan Li ${ }^{1, *}$, Songcheng Yinn ${ }^{2,}{ }^{*}$, Lei Zhang ${ }^{1}$, Weiguang Liu ${ }^{1}$, Bo Chen ${ }^{1}$ \\ ${ }^{1}$ Department of Breast Surgery, The First Hospital of China Medical University, Shenyang, Liaoning 110001, China \\ ${ }^{2}$ Department of Surgical Oncology, The First Hospital of China Medical University, Shenyang, Liaoning 110001, China \\ *These authors contribute equally to this work
}

Correspondence to: Bo Chen, email: chbyxl@163.com

Keywords: E-cadherin, breast cancer, prognosis, biomarker, meta-analysis

Received: July 15, $2016 \quad$ Accepted: January 16, $2017 \quad$ Published: January 27, 2017

\section{ABSTRACT}

The prognostic value of E-cadherin expression in patients with breast cancer has been studied for years, yet results remain controversial. We thus performed a comprehensive evaluation of the association between E-cadherin expression and prognosis through a meta-analysis. The databases PubMed, Embase and Cochrane Library were searched. A total of 7,353 patients from 33 studies were subject to final analysis. The results showed there was a significant association between reduced expression of E-cadherin and overall survival (OS) (HR 1.79, 95\% CI 1.41-2.27) and disease-free survival (DFS) (HR 1.62, 95\% CI 1.31-1.99) in breast cancer. Downregulated expression of E-cadherin significantly correlated with tumor histological grade (OR 1.44, 95\% CI 1.06-1.96), TNM stage (OR 2.44, 95\% CI 1.75-3.41), tumor size (OR 1.38, 95\% CI 1.18-1.60), lymph node status (OR 1.55, 95\% CI 1.15-2.10), and progesterone receptor status (OR 1.44, 95\% CI 1.10-1.88).This meta-analysis suggested that reduced E-cadherin expression might be a predictor of a poorer prognosis and could be a potentially new gene therapy target for breast cancer patients.

\section{INTRODUCTION}

Among women in the world, breast cancer is the most common cancer with an estimated 1.67 million new cases diagnosed ( $25 \%$ of all cancers) and it was the most frequent cause of cancer death (522,000 deaths, $14.7 \%$ of total) in 2012 [1]. Although comprehensive treatment is available,, including radical surgery and adjuvant therapy, the prognosis of breast cancer patients is still far from optimistic [2]. Several common clinicopathological parameters, which include tumor size, lymph node status, histological grade, estrogen receptor (ER), progesterone receptor (PR) and human epidermal growth factor-2 (HER-2) have been extensively applied in the clinic [3]. However, they do not accurately predict an individual's prognosis [4]. It is imperative to explore new prognostic factors to guide treatment and ameliorate survival rates for breast cancer patients [4].

E-cadherin is an essential intercellular adhesion molecule that combines with catenins to form an
E-cadherin/ $\beta$-catenin/ $\alpha$-catenin complex, which is further linked to the actin cytoskeleton [5]. It plays an important role not only in mediating stability of cell adhesion and cell polarity but also in maintaining the integrity of structure and function in epithelial tissues $[6,7]$. Downregulated expression of E-cadherin destroys the intracellular junction and thus epithelial cells acquire the ability to migrate. Consequently, decreased expression of E-cadherin facilitates tumor invasion and metastasis $[8,9]$. Reduced expression of E-cadherin caused by oncocytes involves several molecular mechanisms: $\mathrm{CDH} 1$ gene mutation, $\mathrm{CDH} 1$ promoter hypermethylation, suppression of RNA transcription, and matriptase activation [10].

It has been reported by Rakha et al. that downregulated expression of E-cadherin was correlated with poor survival in a study of 1,516 breast cancer patients [11]. However, Gillett et al. assessed the aberrant expression of E-cadherin in 470 cases of infiltrating ductal cancer (IDC) and concluded that low-expression 
of E-cadherin was a favorable prognostic factor [12]. Wang et al. found there was no relationship between E-cadherin expression and prognostic [13]. Therefore, we conducted a meta-analysis to evaluate the association between E-cadherin low-expression and overall survival (OS), disease-free survival (DFS), and clinicopathological parameters in breast cancer.

\section{RESULTS}

\section{Search results}

A total of 2,192 citations were potentially identified for inclusion using the described search strategies. Through reviewing the title and abstracts, 1,952 papers were excluded. Subsequently, an additional 164 records were excluded for the following reasons: They were reviews, conference abstracts, and experimental studies; the source of the tissue was not breast cancer; and, the target protein was not E-cadherin. We then systematically read the full text of the remaining 76 articles and filtered out an additional 43 papers. Among the excluded papers, 21 studies were not associated with survival, 19 studies had no sufficient survival data to analyze and three studies had overlapped data with other published trials. Ultimately, 33 studies [11-43] were included (Figure 1).

\section{Study characteristics}

The fundamental features of the identified articles are shown in Table 1. The total number of patients was 7,353 , ranging from 29 to 1,516 in any one study with mean ages of 46-60 years. These studies were published between 1994 and 2016. For the prognostic indicator of reduced E-cadherin expression in breast cancer, 15 articles reported both OS and DFS, 10 articles reported OS, and eight articles reported DFS.

\section{Impact of reduced E-cadherin expression on OS and DFS}

The overall analysis revealed that E-cadherinnegative breast cancer patients had a higher risk of mortality (pooled HR 1.79, 95\% CI 1.41-2.27, Figure 2) with heterogeneity $\left(\mathrm{I}^{2}=67.3 \%, P<0.001\right)$. To investigate the source of the OS heterogeneity, subgroup analysis and meta-regression were performed according to publication year, study location, HR estimate, IHC scoring criteria, subcellular localization and pathological types (Table 2). In subgroup analysis, the pooled HRs directly extracted from studies and obtained from Kaplan-Meier curves were 1.77 (95\% CI 1.41-2.28) and 1.92 (95\% CI 1.55-2.39), demonstrating that reduced expression of E-cadherin was significantly associated with poor OS. Meta-regression analysis indicated that there was no statistically significant difference among subgroups $(P=0.637)$. When the scoring criteria of IHC was taken into consideration, the pooled HR of E-cadherin expression in percentage group was 2.19 (95\% CI 1.78-2.70), indicating that there was a significant relationship between reduced expression of E-cadherin and poor OS. In meta-regression analysis, results showed that the difference among subgroups was statistically significant $(P=0.024)$. Pooled HRs were $1.57(95 \%$ CI 1.17-2.10) in the membrane E-cadherin expression group and 2.80 (95\% CI 1.92-4.10) in the membrane and cytoplasm E-cadherin co-expression group. Metaregression analysis showed that there was no statistically significant difference between subgroups $(P=0.061)$.

23 studies evaluated the relationship between decreased E-cadherin expression and DFS, the results showed that E-cadherin low-expression predicted poorer disease-free survival (pooled HR 1.62, 95\% CI 1.31-1.99, Figure 3$)$ with significant heterogeneity $\left(\mathrm{I}^{2}=70.9 \%\right.$, $P<0.001)$ of patients with breast cancer. We also conducted subgroup analysis and meta-regression to explain the heterogeneity from six aspects, which are detailed in Table 2. In subgroup analysis, the pooled HRs directly extracted from studies and obtained from KaplanMeier curves were 1.63 (95\% CI 1.40-1.91) and 1.93 (95\% CI 1.59-2.34). Both of them showed that reduced expression of E-cadherin was significantly associated with disease progression. No significant heterogeneity was found in meta-regression analysis $(P=0.485)$. Pooled HRs were $2.11(95 \%$ CI 1.52-2.92) in the percentage group and 1.47 (95\% CI 1.27-1.70) in the complex score group. Meta-regression analysis showed that no significant statistical difference was found $(P=0.423)$. The results showed that in the group of membrane location (pooled HR 1.37, 95\% CI 1.07-1.75) and group of membrane and cytoplasm location (pooled HR 3.35, 95\% CI 2.03-5.53), indicating that downregulated expression of E-cadherin was correlated with poor DFS. Importantly, a significant heterogeneity was observed in meta-regression analysis $(P=0.031)$.

\section{Evaluation of reduced E-cadherin expression and clinicopathological characteristics}

As illustrated in Table 3, E-cadherin low-expression was significantly associated with lymph node (positive vs. negative: OR $1.55,95 \%$ CI $1.15-2.10)$, tumor size ( $\geq$ $2 \mathrm{~cm}$ vs. $<2 \mathrm{~cm}$, OR 1.38, 95\% CI 1.18-1.60), histological grade (II-III vs. I: OR 1.44, 95\% CI 1.06-1.96), TNM stage (T3/T4 vs. T1/T2: OR 2.44, 95\% CI 1.75-3.41), and PR status (negative vs. positive: OR 1.44, 95\% CI 1.10-1.88). However, no significant correlation was found between E-cadherin low-expression and ER status (negative vs. positive: OR 1.32, 95\% CI 0.94-1.84), HER-2 status ( $\geq 2+$ vs. $1+$ OR $1.36,95 \%$ CI $0.86-2.16$ ), onset age ( $\geq 50$ vs. $<50$ OR 1.03, 95\% CI 0.85-1.24), menstrual status (post vs. premenstrual OR 1.20,95\% CI 0.90-1.60), and pathological type (IDC vs. others OR $0.77,95 \%$ CI $0.59-1.00$ ). 


\section{Sensitivity analysis}

We further performed sensitivity analysis to gauge the stability of our results with respect to OS, DFS, and clinicopathological characteristics. The plots illustrated the robustness of our results because excluding any single study did not significantly influence pooled HRs or ORs (Figure 4).

\section{Publication bias}

To assess the publication bias in this meta-analysis, we used both Egger's test and Begg's funnel plots. Both of these tests present the potential proof of the asymmetry of investigating the reduced expression of E-cadherin on OS $\left(\mathrm{P}_{\text {Egger }}=0.001, \mathrm{P}_{\text {Begg }}=0.388\right)$. Trim-and-fill analysis showed that after incorporating six additional articles, the funnel plots were symmetrical and E-cadherin low-expression was positively correlated with poor OS (corrected HR 1.50, 95\% CI 1.20-1.87). Meanwhile, the impact of absent E-cadherin expression on clinicopathological characteristics, Egger's test indicated publication bias existed in lymph node metastasis $\left(\mathrm{P}_{\text {Egger }}=0.048\right)$. Trim-and-fill analysis was conducted and the results showed that abnormal expression of E-cadherin was significantly correlated with lymph node metastasis after incorporating two additional articles (corrected OR 1.39, 95\% CI 1.04-1.87). Referring to other indicators, no publication bias was found in these articles. Funnel plots are shown in Figure 5.

\section{DISCUSSION}

E-cadherin, a member of cadherin superfamily of transmembrane glycoproteins, is a linker protein of cell-cell junctions [44]. It is well-known that the functional loss of E-cadherin has been viewed as the most important hallmark of epithelial-mesenchymal transition (EMT), which induces tumor cell dissemination and subsequently increases cell migration and invasion [45, 46]. Besides, the absent of E-cadherin expression has an inseparable relationship with resistance of tumor cells to chemotherapy and radiotherapy [47] and causes cancer cells to present apparent properties of cancer stem cells (CSCs) [48]. Many studies have evaluated the association between decreased E-cadherin expression and the prognosis of breast cancer patients. However, the results

\begin{tabular}{|c|c|}
\hline $\begin{array}{l}\text { Records identified through multiple } \\
\text { databases }(\mathrm{N}=2192)\end{array}$ & \\
\hline & $\begin{array}{l}\text { Irrelevant articles excluded by } \\
\text { browsing the titles and abstracts } \\
(\mathrm{N}=1952)\end{array}$ \\
\hline $\begin{array}{l}\text { Records retrieved for further } \\
\text { assessment }(\mathrm{N}=240)\end{array}$ & 164 records excluded \\
\hline & $\begin{array}{l}\text { Reviews }(\mathrm{N}=36) \\
\text { Conference abstracts }(\mathrm{N}=31)\end{array}$ \\
\hline & $\begin{array}{l}\text { Experimental studies }(\mathrm{N}=24) \\
\text { Non-breast cancer diseases }(\mathrm{N}=48)\end{array}$ \\
\hline $\begin{array}{l}\text { Full-text literatures for detailed } \\
\text { evaluation }(\mathrm{N}=76)\end{array}$ & Not target proteins $(\mathrm{N}=25)$ \\
\hline & 43 records excluded \\
\hline & \\
\hline Studies included in this meta-analysis & $\begin{array}{l}\text { Unavailable data }(\mathrm{N}=19) \\
\text { Overlapping data }(\mathrm{N}=3)\end{array}$ \\
\hline
\end{tabular}

Figure 1: Flow diagram of the study selection process. 
Table 1: Characteristics of studies included in our meta-analysis

\begin{tabular}{|c|c|c|c|c|c|c|c|c|c|c|c|c|}
\hline Author & year & country & $\begin{array}{l}\text { Mean age } \\
\text { (year) }\end{array}$ & stage & $\mathbf{N}$ & location & $\begin{array}{l}\text { Median Follow-up } \\
\text { (month) }\end{array}$ & $\begin{array}{l}\text { Cut-off } \\
\text { value }\end{array}$ & Method & Survival & HR estimated & NOS \\
\hline Asgeirsson & 2000 & Iceland & 58 & NR & 108 & $\mathrm{M}$ & 71 & $50 \%$ & $\mathrm{IHC}$ & DFS & HR & 9 \\
\hline Yu & 2015 & China & NR & I-III & 169 & $\mathrm{M}, \mathrm{C}$ & 63.5 & 165 & $\mathrm{IHC}$ & DFS, OS & Curves & 7 \\
\hline Pedersen & 2002 & Norway & 55 & I-IV & 61 & M & 79 & $5 \%$ & $\mathrm{IHC}$ & OS & Curves & 7 \\
\hline Siitonen & 1995 & Finland & 60 & I-IV & 109 & NR & 51 & $10 \%$ & $\mathrm{IHC}$ & DFS & A & 7 \\
\hline Charpin & 1998 & France & 55 & I-III & 179 & M & 67 & $4 \%$ & $\mathrm{IHC}$ & OS & Curves & 8 \\
\hline Kashiwagi & 2010 & Japan & 58 & I-III & 574 & M & 45.7 & $30 \%$ & $\mathrm{IHC}$ & DFS, OS & Curves & 7 \\
\hline Wang & 2015 & China & 47 & I-III & 571 & M & 54 & Scores $\leq 99$ & $\mathrm{IHC}$ & DFS, OS & A & 8 \\
\hline Shi & 2015 & China & 51 & NR & 96 & $\mathrm{M}, \mathrm{C}$ & 65.2 & $28 \%$ & IHC & OS & HR & 9 \\
\hline Pang & 2013 & China & 46 & I-III & 170 & M & 75 & Scores $\leq 3$ & $\mathrm{IHC}$ & DFS, OS & HR & 8 \\
\hline Wang & 2014 & China & 54 & I-III & 29 & NR & 50 & $25 \%$ & $\mathrm{IHC}$ & DFS, OS & A & 8 \\
\hline Liu & 2014 & china & 51 & NR & 100 & C & 65.4 & $28 \%$ & $\mathrm{IHC}$ & OS & HR & 8 \\
\hline Yang & 2015 & China & NR & NR & 125 & M & 89 & Scores $<6$ & IHC & DFS, OS & HR & 7 \\
\hline Bankfalvi & 1999 & Germany & NR & I-IV & 55 & M & 7 & $75 \%$ & IHC & DFS, OS & Curves & 7 \\
\hline Heimann & 2000 & America & 57 & NR & 168 & NR & 168 & $25 \%$ & $\mathrm{IHC}$ & DFS & HR & 9 \\
\hline Pistelli & 2014 & Italy & 54 & I-III & 81 & M & 52.4 & $30 \%$ & IHC & DFS, OS & HR & 8 \\
\hline Gillett & 2001 & UK & 53 & III & 470 & $\mathrm{M}, \mathrm{C}$ & NR & Scores $\leq 1$ & IHC & DFS, OS & A & 6 \\
\hline Kim & 2010 & Korea & 49 & I-IV & 98 & $\mathrm{M}, \mathrm{C}$ & 67.8 & $70 \%$ & $\mathrm{IHC}$ & OS & HR & 7 \\
\hline Lipponen & 1994 & Finland & 57 & I-IV & 207 & M & 171.6 & $50 \%$ & IHC & OS & Curves & 6 \\
\hline Zhou & 2016 & China & NR & I-IV & 119 & $\mathrm{M}, \mathrm{C}$ & 60 & $10 \%$ & IHC & DFS, OS & Curves & 7 \\
\hline $\mathrm{Li}$ & 2014 & China & NR & I-III & 250 & NR & 60 & Scores $<3$ & $\mathrm{IHC}$ & DFS & HR & 7 \\
\hline Park & 2007 & Norway & 54 & I-III & 196 & M & 40 & Scores $\leq 3$ & $\mathrm{IHC}$ & DFS & Curves & 7 \\
\hline Ricciardi & 2015 & Italy & 59 & I-IV & 45 & M & NR & $30 \%$ & IHC & OS & HR & 7 \\
\hline Zhang & 2015 & China & 50 & I-III & 408 & NR & 16 & NR & IHC & DFS, OS & A & 7 \\
\hline Rakha & 2005 & UK & 53 & I-III & 1516 & M & 56 & Scores $\leq 1$ & $\mathrm{IHC}$ & DFS, OS & HR & 8 \\
\hline Saadatmand & 2012 & Netherland & 57 & I-IV & 502 & M & 228 & $53 \%$ & IHC & DFS & HR & 8 \\
\hline Szasz & 2011 & Hungary & 60 & I-III & 197 & M & 111 & NR & $\mathrm{IHC}$ & DFS & Curves & 6 \\
\hline Brzozowska & 2012 & Poland & 58 & I-III & 89 & NR & 113.4 & $70 \%$ & IHC & DFS, OS & Curves & 7 \\
\hline Yoshida & 2001 & Japan & 54 & I-IV & 171 & NR & 59.2 & Scores $<1$ & $\mathrm{IHC}$ & DFS, OS & Curves & 7 \\
\hline Eljuga & 2012 & Croatia & NR & I-III & 134 & M & NR & Scores $\leq 2$ & $\mathrm{IHC}$ & OS & Curves & 7 \\
\hline Kavgaci & 2010 & Turkey & 51 & I-III & 76 & M & 93.6 & $10 \%$ & $\mathrm{IHC}$ & DFS, OS & Curves & 8 \\
\hline Lim & 2002 & Korea & 49 & I-III & 128 & M & 58.5 & $70 \%$ & IHC & OS & A & 8 \\
\hline Kawahara & 1997 & Japan & 52 & I-IV & 98 & NR & 27 & Scores $\leq 4$ & IHC & DFS & Curves & 7 \\
\hline Liu & 2006 & China & 49 & I-III & 54 & M & 36.5 & $10 \%$ & IHC & OS & A & 6 \\
\hline
\end{tabular}

NR, not reported; M, membrane; C, cytoplasm; IHC, immunohistochemistry; DFS, disease-free survival; OS, overall survival; HR: hazard ratio; Curves, extrapolated from Kaplan-Meier curves; A, calculated based on the available information; NOS, Newcastle-Ottawa Scale.

are inconsistent. We summarized outcomes from a total of 33 individual studies that included 7,353 breast cancer patients. From this analysis we reached the conclusion that reduced E-cadherin expression significantly predicted poor OS and DFS. Furthermore, the downregulated expression of E-cadherin was correlated with tumor size, lymph node status, TNM stage, and histological grade.

E-cadherin is often split into fragments in the cytoplasm, which in theory its functions would not play an inhibitory effect on EMT [46, 49]. As a result, we further performed a subgroup according to the location of E-cadherin expression. The results showed that E-cadherin expression on membrane was significantly associated with OS (HR 1.57, 95\% CI 1.17-2.10) and DFS (HR 1.37, 95\% CI 1.07-1.75), which demonstrated that the prognostic role of E-cadherin expressed on the membrane is more precise and meaningful. Considering the heterogeneity of intra-tumor, we conduct a subgroup analysis according to the pathological types of breast cancer. In the subgroup of IDC, the pooled HRs showed that reduced expression of E-cadherin was significantly associated with poor OS (HR 1.62, 95\% CI 1.08-2.43) and DFS (HR 1.60, 95\% CI 1.09-2.34). It's necessary to analyze the heterogeneity of inter-tumor cells as the prognostic values of E-cadherin may quite different in breast cancer stem cell (CSC) subpopulations [48]. However, it's a pity that there is no articles explored the correlation between E-cadherin expression in CSCs and prognosis, and more original research need to be conducted at this field in the future.

When pooling survival data on OS and DFS, we observed significant heterogeneity among articles. Consequently, meta-regression analysis was conducted and it suggested that IHC scoring criteria and subcellular localization might be vital variables associated with 
this heterogeneity. In subgroup analysis of IHC scoring criteria, the heterogeneity of E-cadherin expression calculated by percentage group was less than $50 \%$, while the other groups had significant heterogeneity. The potential reason could be that compared with the detection method of staining intensity, assessing the percentage of positive cells was more objective and had more practical clinical implications. In subgroup analysis according to locations of E-cadherin expression, heterogeneity was significant in membrane E-cadherin expression group. It may mainly come from the differences in sample sizes, molecular subtypes, demographic or clinicopathologic data of observational studies.Compared with analyses performed in 2006 [50], the advantages of this meta-analysis were not only that it included more studies and subjects to confirm clinical validity but also that it provided more rigorous evidence to support the results. More importantly, we demonstrated that the prognostic role of E-cadherin expressed on the membrane is more meaningful. Furthermore, we assessed the association between E-cadherin expression and clinicopathological characteristics for breast carcinoma patients.

There were limitations in our meta-analysis. First, different primary antibody sources and antibody dilution ratios can lead to differences in IHC sensitivity. Second, there was no uniform scoring criteria to define E-cadherin positive expression. Furthermore, cut-off values defining reduced E-cadherin expression varied from 5 to $70 \%$ without an optimal threshold. Third, HRs estimated from available data and obtained from Kaplan-Meier curves were less reliable owing to inaccuracies in the calculation of censored data. Fourth, we had to exclude studies that had no statistical significance because it was difficult to obtain specific data with which to calculate HRs. Finally, meaningful results trended to be published in English, whereas negative ones were more likely to be published in native languages.

In conclusion, our meta-analysis suggest that reduced E-cadherin expression was not only significantly associated with poorer OS and DFS but also correlated with clinicopathological characteristics including tumor size, lymph node status, TNM stage, and histological grade

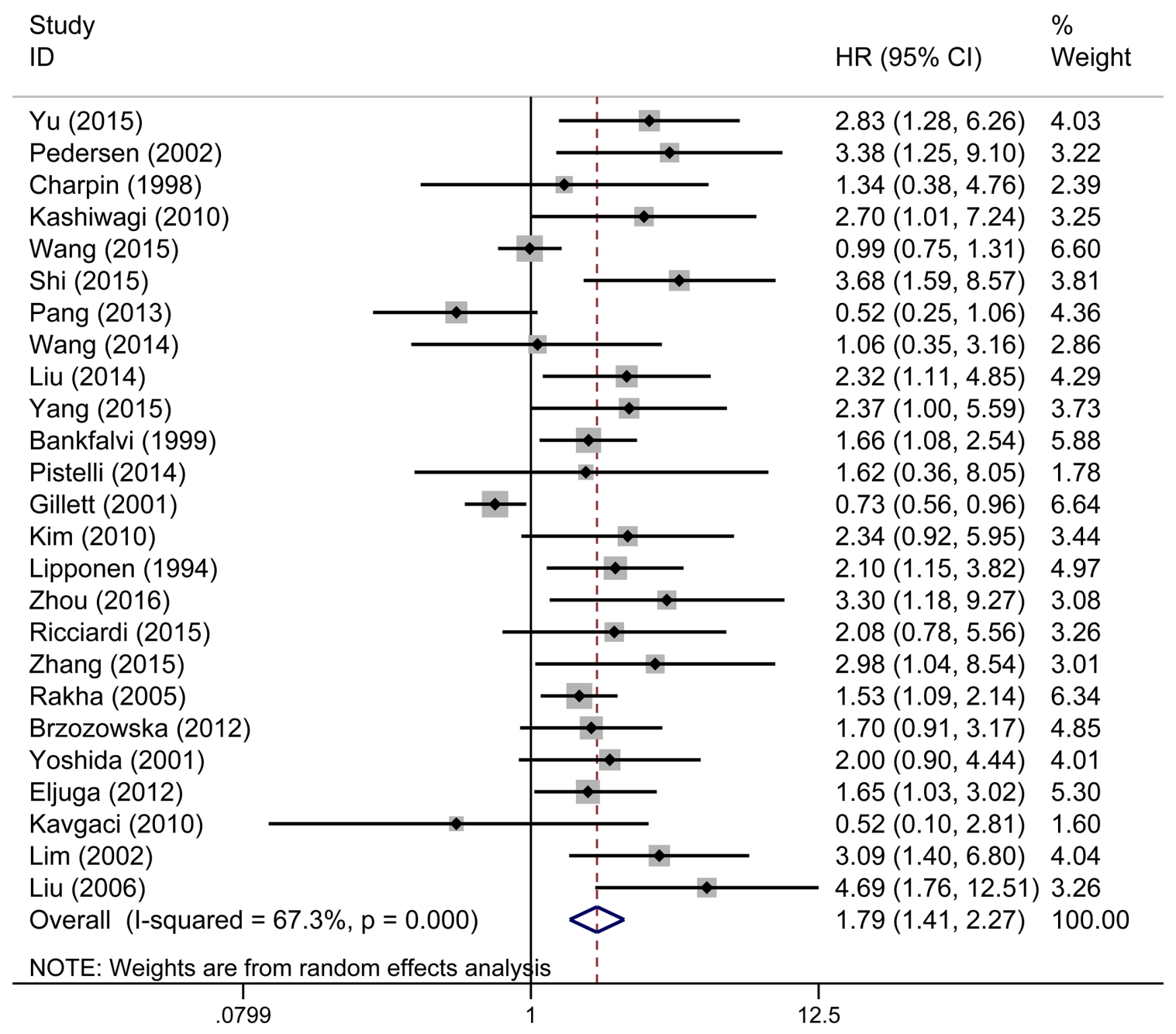

Figure 2: Forest plot of hazard ratio (HR) for the correlation between reduced E-cadherin expression and OS in breast cancer patient. 
Table 2: Stratified analysis of pooled hazard ratios of breast cancer patients with reduced E-cadherin expression on OS and DFS

\begin{tabular}{|c|c|c|c|c|c|c|c|c|c|c|}
\hline \multirow{3}{*}{$\begin{array}{c}\text { Stratified } \\
\text { analysis }\end{array}$} & \multicolumn{5}{|c|}{ OS } & \multicolumn{5}{|c|}{ DFS } \\
\hline & \multicolumn{2}{|c|}{ Pooled HR $(95 \% \mathrm{CI})$} & \multirow{2}{*}{$\begin{array}{c}\text { Meta- } \\
\text { regression } \\
P \text { value }\end{array}$} & \multicolumn{2}{|c|}{ Heterogeneity } & \multicolumn{2}{|c|}{ Pooled HR $(95 \% \mathrm{CI})$} & \multirow{2}{*}{$\begin{array}{l}\text { Meta- } \\
\text { regression } \\
\text { P value }\end{array}$} & \multicolumn{2}{|c|}{ Heterogeneity } \\
\hline & fixed & random & & $I^{2}$ & $P$ value & fixed & random & & $I^{2}$ & $P$ value \\
\hline Year & & & 0.937 & & & & & 0.791 & & \\
\hline$<2010$ & $1.32(1.12,1.56)$ & $1.85(1.22,2.81)$ & & $79.0 \%$ & $<0.001$ & $1.38(1.20,1.59)$ & $1.69(1.19,2.40)$ & & $79.9 \%$ & $<0.001$ \\
\hline$\geq 2010$ & $1.46(1.23,1.73)$ & $1.77(1.30,2.41)$ & & $57.4 \%$ & 0.002 & $1.49(1.29,1.73)$ & $1.58(1.21,1.99)$ & & $63.2 \%$ & 0.001 \\
\hline Nation & & & 0.209 & & & & & 0.925 & & \\
\hline Asia & $1.56(1.30,1.88)$ & $2.12(1.45,3.09)$ & & $68.9 \%$ & $<0.001$ & $1.44(1.21,1.71)$ & $1.61(1.13,2.28)$ & & $69.6 \%$ & $<0.001$ \\
\hline Non-Asia & $1.27(1.08,1.48)$ & $1.51(1.09,2.08)$ & & $65.8 \%$ & 0.001 & $1.43(1.27,1.61)$ & $1.63(1.24,2.14)$ & & $74.3 \%$ & $<0.001$ \\
\hline HR estimate & & & 0.637 & & & & & 0.485 & & \\
\hline Directly & $1.65(1.30,2.09)$ & $1.77(1.41,2.28)$ & & $55.5 \%$ & 0.028 & $1.67(1.23,2.26)$ & $1.63(1.40,1.91)$ & & $62.4 \%$ & 0.009 \\
\hline Calculated & $0.99(0.83,1.19)$ & $1.60(0.93,2.75)$ & & $81.3 \%$ & $<0.001$ & $1.13(0.75,1.71)$ & $0.94(0.79,1.13)$ & & $74.1 \%$ & 0.004 \\
\hline Curves & $1.92(1.55,2.39)$ & $1.92(1.55,2.39)$ & & $0.0 \%$ & 0.693 & $1.93(1.59,2.34)$ & $1.93(1.59,2.34)$ & & $0.0 \%$ & 0.512 \\
\hline Scoring criteria & & & 0.024 & & & & & 0.423 & & \\
\hline Percentage & $2.19(1.78,2.70)$ & $2.19(1.78,2.70)$ & & $0.0 \%$ & 0.613 & $2.11(1.52,2.92)$ & $2.13(1.68,2.70)$ & & $38.5 \%$ & 0.135 \\
\hline Intensity & $0.93(0.74,1.16)$ & $1.21(0.68,2.15)$ & & $72.9 \%$ & 0.011 & $1.19(0.76,1.87)$ & $1.09(0.92,1.30)$ & & $79.6 \%$ & 0.001 \\
\hline Combined & $1.22(1.00,1.48)$ & $1.33(0.84,2.10)$ & & $74.8 \%$ & 0.003 & $1.58(1.20,2.09)$ & $1.47(1.27,1.70)$ & & $63.3 \%$ & 0.004 \\
\hline Location & & & 0.061 & & & & & 0.031 & & \\
\hline M & $1.24(1.09,1.41)$ & $1.57(1.17,2.10)$ & & $71.8 \%$ & $<0.001$ & $1.29(1.16,1.45)$ & $1.37(1.07,1.75)$ & & $73.8 \%$ & $<0.001$ \\
\hline $\mathrm{C}, \mathrm{M}$ & $2.80(1.92,4.10)$ & $2.80(1.92,4.10)$ & & $0 \%$ & 0.925 & $3.35(2.03,5.53)$ & $3.35(2.03,5.53)$ & & $0.0 \%$ & 0.529 \\
\hline \multicolumn{11}{|l|}{ Pathological type } \\
\hline IDC & $1.13(0,97,1.32)$ & $1.61(1.09,2.39)$ & & $78.1 \%$ & $<0.001$ & $1.12(0.95,1.31)$ & $1.58(1.03,2.44)$ & & $82.4 \%$ & $<0.001$ \\
\hline
\end{tabular}

of breast cancer patients. E-cadherin low-expression might be a useful biomarker for predicting poorer prognosis, especially in the location of membrane, and could be a valuable therapeutic target for breast cancer patients.

\section{MATERIALS AND METHODS}

\section{Search strategy}

We searched for studies on E-cadherin expression and its association with breast cancer prognosis in electronic databases: PubMed, EMBASE, and Cochrane library updated to May 15, 2016. Articles were qualified using the following combined keywords: "breast", "mammary", "tumor", "cancer", "neoplasm", "E-cadherin", "E-CAD", "cadherin-1", "prognostic", and "survival". References from eligible literature were scanned to minimize any deviation caused during the research process.

\section{Inclusion criteria}

Articles were required to meet the following inclusion criteria: (1) patients diagnosed with breast cancer using pathological and histological examinations; (2) E-cadherin expression was detected in primary tumor tissues; (3) full text, original research articles published in English; (4) statistical results that included hazard ratios (HRs) and 95\% confidence intervals (CIs) reported directly or calculated from demographic data or survival curves; and (5) independent E-cadherin expression level data. Only studies with more details and larger sample sizes were selected if duplicate data from other articles occurred. Reviews, letters, conference abstracts, and comments were excluded.

\section{Quality assessment}

The Newcastle-Ottawa quality assessment scale (NOS) was applied to estimate the quality of nonrandomized studies, specifically cohort studies by two investigators independently. According to the NOS, three perspectives were assessed: selection, comparability, and outcomes. Scores higher than six were considered high quality.

\section{Data extraction}

Two researchers extracted the following data independently from qualified studies: (1) publication data including authors, year and country; (2) experimental data including tissue origin, location of E-cadherin expression, percentage of E-cadherin positive cells and cut-off values; (3) demographic data including number of subjects analyzed, ages, and follow-ups; (4) clinicopathological data including tumor size, lymph node status, ER, PR, HER-2, menopausal state, 
Table 3: Meta-analysis of reduced E-cadherin expression and clinicopathological features in breast cancer

\begin{tabular}{lcccccc}
\hline & $\begin{array}{c}\text { No. of } \\
\text { studies }\end{array}$ & Pheterogeneity & $\begin{array}{c}\mathbf{I}^{\mathbf{2}} \\
(\mathbf{\%})\end{array}$ & Effect Model & $\begin{array}{c}\text { Pooled } \\
\text { OR(95\%CI) }\end{array}$ & $\begin{array}{c}\boldsymbol{P} \\
\text { Value }\end{array}$ \\
\hline Tumor size( $\geq$ 2 vs. $<2)$ & 12 & 0.734 & 0.0 & Fixed model & $1.38(1.18,1.60)$ & $<0.001$ \\
Age( $\geq$ 50 vs. $<50)$ & 12 & 0.205 & 25.0 & Fixed model & $1.03(0.85,1.24)$ & 0.706 \\
Histological grade(II/IIIvs.I) & 14 & 0.001 & 63.6 & Random model & $1.44(1.06,1.96)$ & 0.02 \\
TNM stage(T3/T4vs.T1/T2) & 7 & 0.086 & 45.9 & Fixed model & $2.44(1.75,3.41)$ & $<0.001$ \\
Pathological type(IDC vs.Others) & 7 & 0.629 & 0.0 & Fixed model & $0.77(0.59,1.00)$ & 0.054 \\
Menopause status(Post vs.Pre) & 6 & 0.341 & 11.6 & Fixed model & $1.20(0.90,1.60)$ & 0.219 \\
Lymph node status(+ vs.- $)$ & 15 & $<0.001$ & 72.1 & Random model & $1.55(1.15,2.10)$ & 0.005 \\
ER status(- vs.+) & 12 & 0.002 & 61.8 & Random model & $1.32(0.94,1.84)$ & 0.108 \\
PR status $(-$ vs.+) & 8 & 0.399 & 4.0 & Fixed model & $1.44(1.10,1.88)$ & 0.007 \\
Her2 status( $\geq 2+$ vs.1+) & 5 & 0.197 & 33.6 & Fixed model & $1.36(0.86,2.16)$ & 0.185 \\
\hline
\end{tabular}

Study
ID

Figure 3: A. Forest plot of hazard ratio (HR) for the association between reduced E-cadherin expression and DFS in breast cancer patient. 
pathologic type, histologic type, and TNM stage; and (5) statistical data including survival analysis, HRs and 95\% CIs. Inconsistencies were resolved through negotiation and consultation.

\section{Statistical analysis}

In this meta-analysis, HRs with 95\% CIs was applied as appropriate values to measure the impact of reduced E-cadherin expression on survival in breast cancer. In some studies, the value of HR and the $95 \%$ CI describing OS and/or DFS could be obtained directly. Otherwise, many studies displaying survival rates with $P$ values from log-rank tests or Kaplan-Meier survival curves could also be extrapolated using the method of Parmar and Tierney [51]. For the pooled analysis of the correlation between decreased E-cadherin expression and clinicopathological features, odds ratios (ORs) and

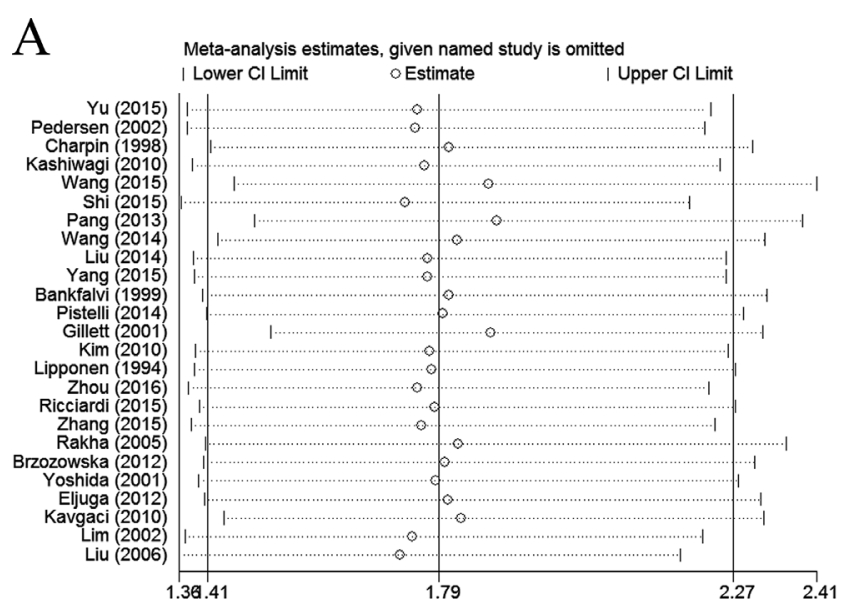

their 95\% CIs were evaluated. Heterogeneity of the studies was evaluated by the Chi-square-based $Q$ test and $\mathrm{I}^{2}$. $\mathrm{I}^{2}<50 \%$ and $P>0.05$ were considered as acceptable heterogeneity, in which case the fixed-effect model test was performed. Otherwise, the random-effect model test was chosen if significant heterogeneity existed $\left(\mathrm{I}^{2}>50 \%\right.$ or $\left.P<0.05\right)$. Subgroup analysis and metaregression analysis were performed to detect the source of the heterogeneity. Publication bias was assessed using Begg's test and Egger's test. Sensitivity analysis was carried out to evaluate the stability of the pooled results using sequential omission of individual studies. Furthermore, if multivariate and univariate analyses were both obtainable, the former was chosen. All $P$ values were two-sided and $P<0.05$ was considered statistically significant. All statistical analyses were performed with Stata Version 12.0 (Stata Corporation, College Station, TX, USA).

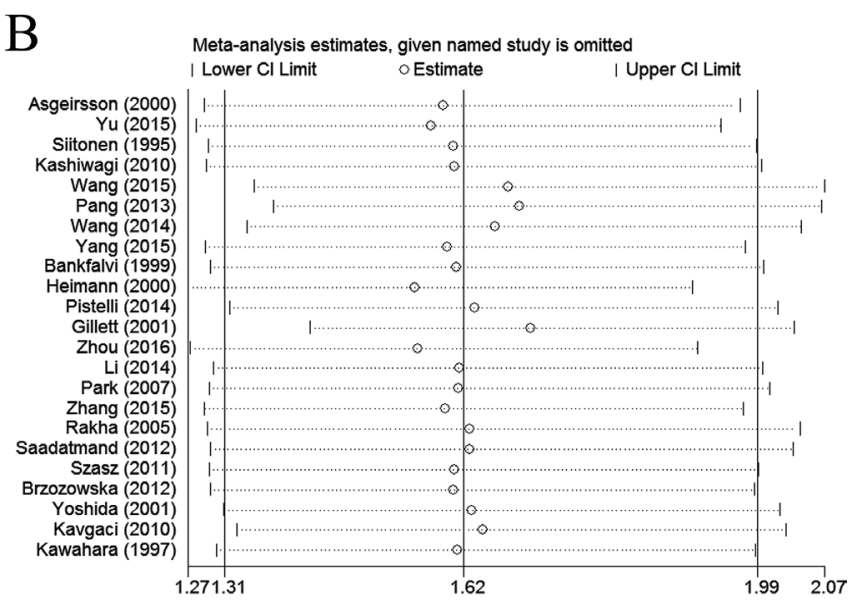

Figure 4: Sensitivity analysis in this meta-analysis. (A) Sensitivity analysis for the reduced E-cadherin expression with OS. (B) Sensitivity analysis for the reduced E-cadherin expression with DFS.
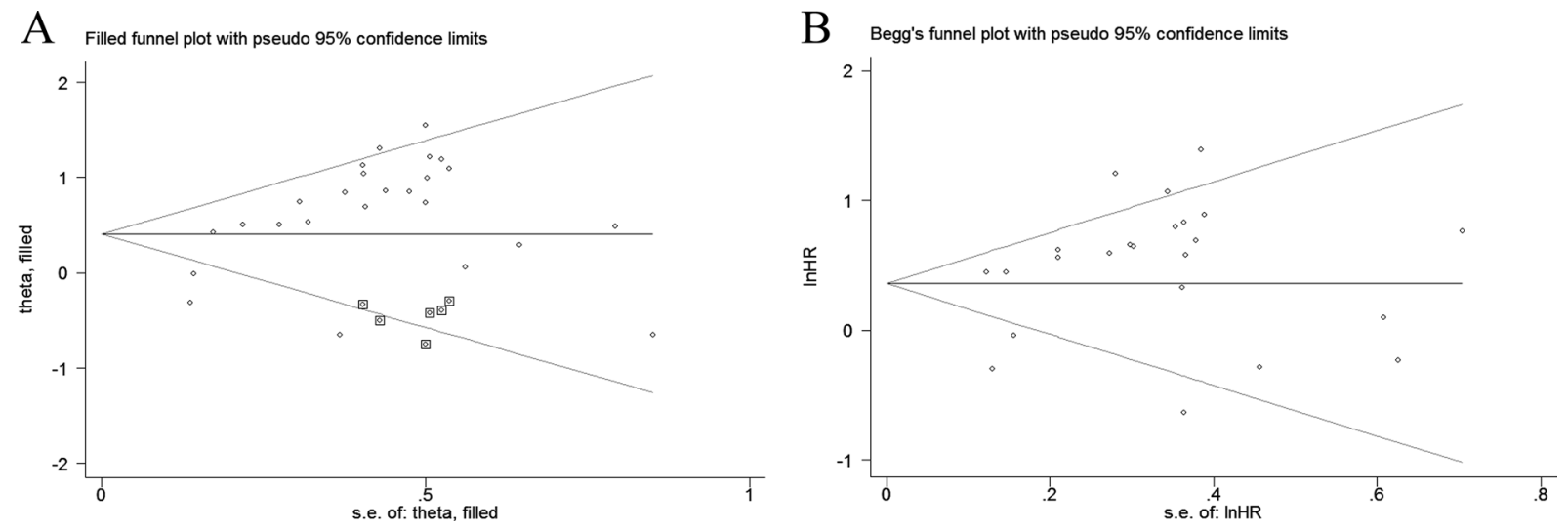

Figure 5: Funnel plot for the assessment of publication bias in this study. (A) Funnel plot of trim-and-fill analysis for the reduced E-cadherin expression with OS (B) Funnel plot for the reduced E-cadherin expression with DFS. 


\section{ACKNOWLEDGMENTS AND FUNDING}

This study was supported by the grants from the National Natural Science Foundation of China (No. 81372811) and Science and Technology Agency of Liaoning Province (No. 2013225049).

\section{CONFLICTS OF INTEREST}

No conflicts of interest exist for any authors.

\section{REFERENCES}

1. Ferlay J, Soerjomataram I, Dikshit R, Eser S, Mathers C, Rebelo M, Parkin DM, Forman D, Bray F. Cancer incidence and mortality worldwide: sources, methods and major patterns in GLOBOCAN 2012. Int J Cancer. 2015; 136:E359-86. doi: 10.1002/ijc.29210.

2. Xu C, Wei Q, Guo J, Zhou JC, Mei J, Jiang ZN, Shen JG, Wang LB. FOXA1 Expression Significantly Predict Response to Chemotherapy in Estrogen Receptor-Positive Breast Cancer Patients. Ann Surg Oncol. 2015; 22:2034-9. doi: 10.1245/s10434-014-4313-2.

3. Rakha EA, Reis-Filho JS, Ellis IO. Combinatorial biomarker expression in breast cancer. Breast Cancer Res Treat. 2010; 120:293-308. doi: 10.1007/s10549-010-0746-x.

4. Donegan WL. Tumor-related prognostic factors for breast cancer. CA Cancer J Clin. 1997; 47:28-51.

5. Zhao Z, Ge J, Sun Y, Tian L, Lu J, Liu M, Zhao Y. Is E-cadherin immunoexpression a prognostic factor for head and neck squamous cell carcinoma (HNSCC)? A systematic review and meta-analysis. Oral Oncol. 2012; 48:761-7. doi: 10.1016/j.oraloncology.2012.02.024.

6. Luo SL, Xie YG, Li Z, Ma JH, Xu X. E-cadherin expression and prognosis of oral cancer: a meta-analysis. Tumour Biol. 2014; 35:5533-7. doi: 10.1007/s13277-014-1728-0.

7. Ren ZH, Xu JL, Li B, Fan TF, Ji T, Zhang CP. Elective versus therapeutic neck dissection in node-negative oral cancer: Evidence from five randomized controlled trials. Oral Oncol. 2015; 51:976-81. doi: 10.1016/j. oraloncology.2015.08.009.

8. Takeichi M. Cadherin cell adhesion receptors as a morphogenetic regulator. Science. 1991; 251:451-5.

9. Deeb G, Wang J, Ramnath N, Slocum HK, Wiseman S, Beck A, Tan D. Altered E-cadherin and epidermal growth factor receptor expressions are associated with patient survival in lung cancer: a study utilizing high-density tissue microarray and immunohistochemistry. Mod Pathol. 2004; 17:430-9. doi: 10.1038/modpathol.3800041.

10. Goodwin M, Yap AS. Classical cadherin adhesion molecules: coordinating cell adhesion, signaling and the cytoskeleton. J Mol Histol. 2004; 35:839-44. doi: 10.1007/ s10735-004-1833-2.
11. Rakha EA, Abd El Rehim D, Pinder SE, Lewis SA, Ellis IO. E-cadherin expression in invasive non-lobular carcinoma of the breast and its prognostic significance. Histopathology. 2005; 46:685-93. doi: 10.1111/j.1365-2559.2005.02156.x.

12. Gillett CE, Miles DW, Ryder K, Skilton D, Liebman RD, Springall RJ, Barnes DM, Hanby AM. Retention of the expression of E-cadherin and catenins is associated with shorter survival in grade III ductal carcinoma of the breast. J Pathol. 2001; 193:433-41. doi: 10.1002/path.831.

13. Wang J, Zhang C, Chen K, Tang H, Tang J, Song C, Xie X. ERbetal inversely correlates with PTEN/PI3K/ AKT pathway and predicts a favorable prognosis in triplenegative breast cancer. Breast Cancer Res Treat. 2015; 152:255-69. doi: 10.1007/s10549-015-3467-3.

14. Asgeirsson KS, Jonasson JG, Tryggvadottir L, Olafsdottir K, Sigurgeirsdottir JR, Ingvarsson S, Ogmundsdottir HM. Altered expression of E-cadherin in breast cancer. patterns, mechanisms and clinical significance. Eur J Cancer. 2000; 36:1098-106.

15. Yu Z, Sun M, Jin F, Xiao Q, He M, Wu H, Ren J, Zhao L, Zhao H, Yao W, Shan F, Cao Y, Wei M. Combined expression of ezrin and E-cadherin is associated with lymph node metastasis and poor prognosis in breast cancer. Oncol Rep. 2015; 34:165-74. doi: 10.3892/or.2015.3967.

16. Pedersen KB, Nesland JM, Fodstad O, Maelandsmo GM. Expression of S100A4, E-cadherin, alpha- and beta-catenin in breast cancer biopsies. Br J Cancer. 2002; 87:1281-6. doi: $10.1038 /$ sj.bjc.6600624.

17. Siitonen SM, Kononen JT, Helin HJ, Rantala IS, Holli KA, Isola JJ. Reduced E-cadherin expression is associated with invasiveness and unfavorable prognosis in breast cancer. Am J Clin Pathol. 1996; 105:394-402.

18. Charpin C, Garcia S, Bonnier P, Martini F, Andrac L, Choux R, Lavaut MN, Allasia C. Reduced E-cadherin immunohistochemical expression in node-negative breast carcinomas correlates with 10-year survival. Am J Clin Pathol. 1998; 109:431-8.

19. Kashiwagi $S$, Yashiro $M$, Takashima $T$, Nomura $S$, Noda S, Kawajiri H, Ishikawa T, Wakasa K, Hirakawa K. Significance of E-cadherin expression in triple-negative breast cancer. Br J Cancer. 2010; 103:249-55. doi: 10.1038/ sj.bjc.6605735.

20. Shi Y, Liu X, Sun Y, Wu D, Qiu A, Cheng H, Wu C, Wang $\mathrm{X}$. Decreased expression and prognostic role of EHD2 in human breast carcinoma: correlation with E-cadherin. J Mol Histol. 2015; 46: 221-31. doi: 10.1007/ s10735-015-9614-7.

21. Pang H, Lu H, Song H, Meng Q, Zhao Y, Liu N, Lan F, Liu Y, Yan S, Dong X, Cai L. Prognostic values of osteopontin-c, E-cadherin and beta-catenin in breast cancer. Cancer Epidemiol. 2013; 37:985-92. doi: 10.1016/j. canep.2013.08.005. 
22. Wang J, Zhang X, He J, Yang M, Tang J, Li X, Tang H, Xie X. Co-expression of EGFR and CK5/6 in primary squamous cell carcinoma of the breast. Med Oncol. 2014; 31:172. doi: 10.1007/s12032-0 14-0172-8.

23. Liu X, Yao N, Qian J, Huang H. High expression and prognostic role of CAP1 and CtBP2 in breast carcinoma: associated with E-cadherin and cell proliferation. Med Oncol. 2014; 31:878. doi: 10.1007/s12032-014-0878-7.

24. Yang Z, Zhang B, Liu B, Xie Y, Cao X. Combined Runx2 and Snail overexpression is associated with a poor prognosis in breast cancer. Tumour Biol. 2015; 36:4565-73. doi: 10.1007/s13277-015-3101-3.

25. Bankfalvi A, Terpe HJ, Breukelmann D, Bier B, Rempe D, Pschadka G, Krech R, Lelle RJ, Boecker W. Immunophenotypic and prognostic analysis of E-cadherin and beta-catenin expression during breast carcinogenesis and tumour progression: a comparative study with CD44. Histopathology. 1999; 34:25-34.

26. Heimann R, Lan F, McBride R, Hellman S. Separating favorable from unfavorable prognostic markers in breast cancer: the role of E-cadherin. Cancer Res. 2000; 60: 298-304.

27. Pistelli M, Caramanti M, Biscotti T, Santinelli A, Pagliacci A, De Lisa M, Ballatore Z, Ridolfi F, Maccaroni E, Bracci R, Berardi R, Battelli N, Cascinu S. Androgen receptor expression in early triple-negative breast cancer: clinical significance and prognostic associations. Cancers (Basel). 2014; 6:1351-62. doi: 10.3390/cancers6031351.

28. Kim HS, Kim GY, Kim YW, Park YK, Song JY, Lim SJ. Stromal CD10 expression and relationship to the E-cadherin/beta-catenin complex in breast carcinoma. Histopathology. 2010; 56:708-19. doi: 10.1111/j.13652559.2010.03534.x.

29. Lipponen P, Saarelainen E, Ji H, Aaltomaa S, Syrjanen K. Expression of E-cadherin (E-CD) as related to other prognostic factors and survival in breast cancer. J Pathol. 1994; 174:101-9. doi: 10.1002/path.1711740206.

30. Zhou D, Zhang M, Xu P, Yu Y, Ye G, Zhang L, Wu A. Expression of pigment epithelium-derived factor is associated with a good prognosis and is correlated with epithelial-mesenchymal transition-related genes in infiltrating ductal breast carcinoma. Oncol Lett. 2016; 11:116-24. doi: 10.3892/ol.2015.3880.

31. Li K, Yao L, Chen L, Cao ZG, Yu SJ, Kuang XY, Hu X, Shao ZM. ID2 predicts poor prognosis in breast cancer, especially in triple-negative breast cancer, and inhibits E-cadherin expression. Onco Targets Ther. 2014; 7: 1083-94. doi: 10.2147/ott.s64759.

32. Park D, Karesen R, Axcrona U, Noren T, Sauer T. Expression pattern of adhesion molecules (E-cadherin, alpha-, beta-, gamma-catenin and claudin-7), their influence on survival in primary breast carcinoma, and their corresponding axillary lymph node metastasis. Apmis. 2007; 115:52-65. doi: 10.1111/j.1600-0463.2007. apm_524.x.
33. Ricciardi GR, Adamo B, Ieni A, Licata L, Cardia R, Ferraro G, Franchina T, Tuccari G, Adamo V. Androgen Receptor (AR), E-Cadherin, and Ki-67 as Emerging Targets and Novel Prognostic Markers in Triple-Negative Breast Cancer (TNBC) Patients. PLoS One. 2015; 10:e0128368. doi: 10.1371/journal.pone.0128368.

34. Zhang YQ, Wei XL, Liang YK, Chen WL, Zhang F, Bai JW, Qiu SQ, Du CW, Huang WH, Zhang GJ. OverExpressed Twist Associates with Markers of Epithelial Mesenchymal Transition and Predicts Poor Prognosis in Breast Cancers via ERK and Akt Activation. PLoS One. 2015; 10:e0135851. doi: 10.1371/journal.pone.0135851.

35. Saadatmand S, de Kruijf EM, Sajet A, Dekker-Ensink NG, van Nes JG, Putter H, Smit VT, van de Velde CJ, Liefers GJ, Kuppen PJ. Expression of cell adhesion molecules and prognosis in breast cancer. Br J Surg. 2013; 100:252-60. doi: $10.1002 /$ bjs. 8980 .

36. Szasz AM, Nemeth Z, Gyorffy B, Micsinai M, Krenacs T, Baranyai Z, Harsanyi L, Kiss A, Schaff Z, Tokes AM, Kulka J. Identification of a claudin-4 and E-cadherin score to predict prognosis in breast cancer. Cancer Sci. 2011; 102: 2248-54. doi: 10.1111/j.1349-7006.2011.02085.x.

37. Brzozowska A, Sodolski T, Duma D, Mazurkiewicz T, Mazurkiewicz M. Evaluation of prognostic parameters of E-cadherin status in breast cancer treatment. Ann Agric Environ Med. 2012; 19:541-6.

38. Yoshida R, Kimura N, Harada Y, Ohuchi N. The loss of E-cadherin, alpha- and beta-catenin expression is associated with metastasis and poor prognosis in invasive breast cancer. Int J Oncol. 2001; 18:513-0.

39. Eljuga D, Bulic K, Petrovecki M, Bali V, Ozimec E, Razumovic JJ. Reduced E-cadherin expression is a predictor of lower overall survival and metastatic disease in invasive ductal breast cancer. Onkologie. 2012; 35:414-8. doi: $10.1159 / 000341071$.

40. Kavgaci H, Yildiz B, Fidan E, Reis A, Ozdemir F, Cobanoglu U, Can G. The effects of E-cadherin and bcl2 on prognosis in patients with breast cancer. Bratisl Lek Listy. 2010; 111:493-7.

41. Lim SC, Lee MS. Significance of E-cadherin/beta-catenin complex and cyclin D1 in breast cancer. Oncol Rep. 2002; 9:915-28.

42. Kawahara F, Noguchi M, Yagasaki R, Minami M, Earashi M, Kinoshita K, Ohta N, Taniya T, Miyazaki I. The expression of E-cadherin as a prognostic factor in breast cancer. Oncol Rep. 1997; 4:401-5.

43. Liu Z, Cui DX, Liu BL, Zhang XB, Ma WF, Zhang Q. Expression of E-cadherin/catenin complex in breast cancer. Chinese Journal of Cancer Research. 2006; 18:299-305.

44. Gumbiner BM. Cell adhesion: the molecular basis of tissue architecture and morphogenesis. Cell. 1996; 84:345-57.

45. Ashaie MA, Chowdhury EH. Cadherins: The Superfamily Critically Involved in Breast Cancer. Curr Pharm Des. 2016; 22:616-38. 
46. Zhang Y, Zhao Y, Jiang G, Zhang X, Zhao H, Wu J, Xu K, Wang E. Impact of p120-catenin isoforms $1 \mathrm{~A}$ and $3 \mathrm{~A}$ on epithelial mesenchymal transition of lung cancer cells expressing E-cadherin in different subcellular locations. PLoS One. 2014; 9:e88064. doi: 10.1371/journal. pone. 0088064 .

47. Nantajit D, Lin D, Li JJ. The network of epithelialmesenchymal transition: potential new targets for tumor resistance. J Cancer Res Clin Oncol. 2015; 141:1697-13. doi: 10.1007/s00432-014-1840-y.

48. Kong D, Li Y, Wang Z, Sarkar FH. Cancer Stem Cells and Epithelial-to-Mesenchymal Transition (EMT)-Phenotypic Cells: Are They Cousins or Twins? Cancers (Basel). 2011; 3:716-29. doi: 10.3390/cancers30100716.
49. Du W, Liu X, Fan G, Zhao X, Sun Y, Wang T, Zhao R, Wang G, Zhao C, Zhu Y, Ye F, Jin X, Zhang F, et al. From cell membrane to the nucleus: an emerging role of E-cadherin in gene transcriptional regulation. J Cell Mol Med. 2014; 18:1712-9. doi: 10.1111/jcmm. 12340.

50. Ihara A, Koizumi H, Hashizume R, Uchikoshi T. Expression of epithelial cadherin and alpha- and beta-catenins in nontumoral livers and hepatocellular carcinomas. Hepatology. 1996; 23:1441-7. doi: 10.1053/jhep.1996.v23. pm0008675162.

51. Parmar MK, Torri V, Stewart L. Extracting summary statistics to perform meta-analyses of the published literature for survival endpoints. Stat Med. 1998; 17: 2815-34. 